\title{
Delayed Boston Keratoprosthesis Exchange due to a Preceding Vitreoretinal Surgery with Intraoperative Choroidal Detachment
}

\author{
María Isabel Relimpio López María Gessa Sorroche \\ Antonio Manuel Garrido Hermosilla Teresa Laborda Guirao \\ Francisco Espejo Arjona Enrique Rodríguez de la Rúa Franch \\ Virgin Macarena University Hospital, Seville, Spain
}

\begin{abstract}
Purpose: The aim is to describe the main characteristics of an anterior/posterior segment surgery and how to resolve intraoperative complications. Setting/Venue: The anterior and posterior segment surgical video was created at the Department of Ophthalmology, Virgin Macarena University Hospital, Seville, Spain. Methods: We present the case of a male with Stevens-Johnson syndrome and severe limbal deficiency who needed a Boston type 1 keratoprosthesis, reaching a visual acuity of 0.4 (0.05 before surgery). In the course of follow-up, he developed corneal melting with perforation, immune vitritis, and a large epimacular membrane. We decided to perform a 23-gauge vitrectomy associated with keratoprosthesis exchange. As a consequence of inappropriate anesthesia, the patient woke up during the sur-
\end{abstract}

gery, provoking a retinal tear besides a choroidal detachment. These damages needed endolaser photocoagulation as well as silicone oil tamponade, forcing us to postpone the exchange. An intravitreal dexamethasone implant was also injected. Two months later, the silicone oil was removed, and the Boston keratoprosthesis was replaced by a new type 1 model with a titanium back plate, which likely improves biocompatibility and retention and may reduce complications such as retroprosthetic membranes and stromal corneal melts. Results: Good anatomical results were achieved, and visual acuity slightly improved to 0.2. Conclusions: Combined anterior and posterior segment surgery represents a great challenge that can improve not only visual acuity but also quality of life in patients with severe diseases such as Stevens-Johnson syndrome.

c) 2015 S. Karger AG, Basel

\section{KARGER}

E-Mail karger@karger.com www.karger.com/oph
(C) 2015 S. Karger AG, Basel

0030-3755/15/2351-0061\$39.50/0
María Isabel Relimpio López

Virgin Macarena University Hospital

Avd. Doctor Fedriani 3

ES-41071 Seville (Spain)

irelimpio@gmail.com 